\title{
Real-Time Error Correction for Quantum Computing
}

\author{
An experiment shows that errors in quantum computation can be \\ repeatedly corrected on the fly.
}

By Philip Ball

$\sum^{2}$ andom errors incurred during computation are one of the biggest obstacles to unleashing the full power of quantum computers. Researchers have now demonstrated a technique that allows errors to be detected and corrected in real time as the computation proceeds. It also allows error correction to be conducted several times on a single quantum bit (qubit) during the calculation [1]. Both features are needed to make the basic elements-the logical qubits-of a fully error-tolerant quantum computer that can be scaled up and used for applications beyond the specialized ones that these machines have tackled so far.

Error correction is straightforward on classical computers: by keeping several copies of each bit, a random error (such as a 1 flipping to a 0) can be identified and corrected using a simple majority rule. But that can't be done in quantum computers,

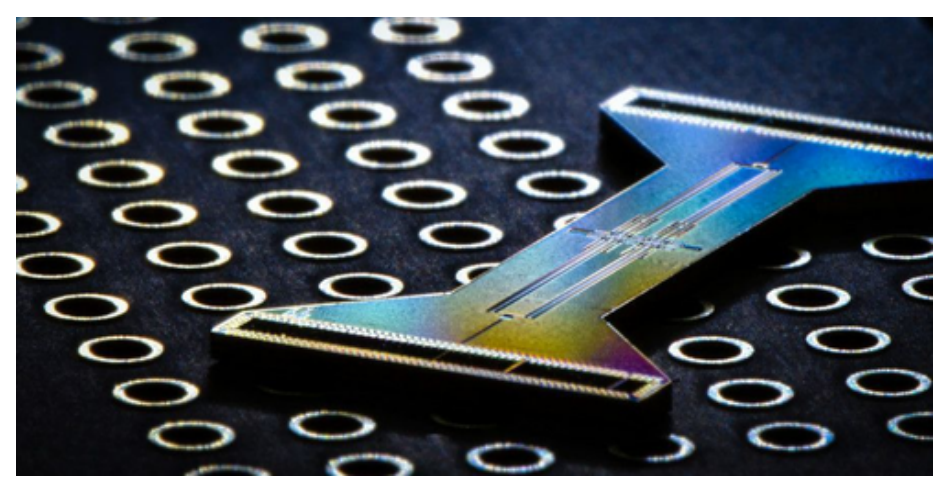

Quantum computation without all the noise. The ion-trap chip used for the error-correction scheme holds ten single-ion qubits lined up in a row.

Credit: Honeywell Quantum Solutions/Quantinuum since the computation relies on the qubits adopting quantum states that are neither 0 nor 1 , and measuring them destroys those delicate states. So the states must remain unknown, and a fundamental principle forbids the copying of an unknown quantum state.

If qubit errors are not corrected, they will gradually accumulate and overwhelm the calculation with random noise, limiting the number of steps a quantum algorithm can reliably perform. The problem gets harder to manage as the number of qubits increases. That's why today's quantum computers have only a relatively small number of "noisy" qubits. The best of these quantum circuits may still outperform classical computers for certain types of calculations, but without quantum error correction (QEC), the power and scope of quantum computing remain limited.

Various QEC methods have been proposed in which several physical qubits are quantum mechanically entangled to make a single logical qubit that has some resistance to errors. But these schemes have typically allowed only one round of error correction for each logical qubit in a calculation. Another problem is that they have generally been retrospective-the final result is "post-corrected"-but not all errors can be corrected after-the-fact.

A team of researchers at Quantinuum (formerly Honeywell Quantum Solutions) in Broomfield, Colorado, has now implemented a method that overcomes some of these limitations. They use a QEC scheme first proposed in 1996 by Andrew Steane of the University of Oxford, UK [2]. Like most other QEC approaches, it involves additional so-called ancilla qubits, whose job is to signal errors in the "data qubits." In each 


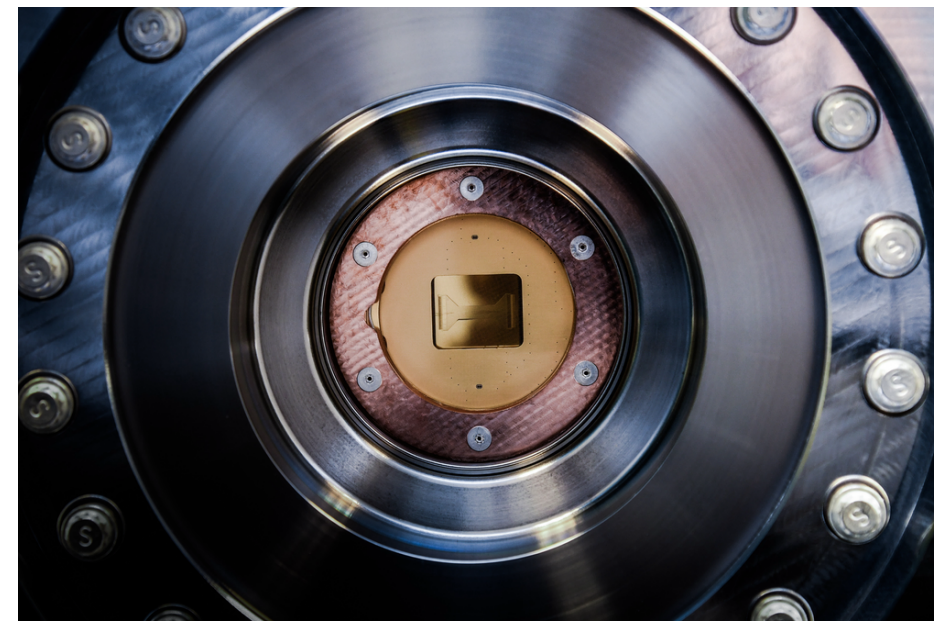

Ready for action. The Quantinuum chip inside its vacuum chamber.

Credit: Honeywell Quantum Solutions/Quantinuum

of a series of operations, an ancilla qubit is entangled with a subset of data qubits, and then the ancilla qubit is measured. This process allows some information about the data qubits to be inferred without measuring them directly.

Although the basic idea has been around since the 1990s, putting it into practice with schemes for rapidly and repeatedly diagnosing and fixing the errors in a real quantum circuit with good-quality qubits posed a massive technological challenge. The algorithms and hardware developed by Ryan-Anderson and his colleagues now address these problems.

The researchers used qubits made from single ytterbium ions held in an electromagnetic trap. The system allows each ion to be moved into proximity with any other ion to enable the entanglement operations. The qubits are embodied in the quantum electronic states of the ions, which can be manipulated by laser beams. To read a qubit, the team hits the ion with a laser pulse and measures its fluorescence. Such trapped-ion quantum circuits are already being developed for commercial quantum computers. Ryan-Anderson and colleagues used ten of these physical-qubit ions to make a single error-tolerant logical qubit: seven of them were data qubits, and the other three were ancilla qubits.

The team showed that they could perform multiple rounds of error detection and correction on each data qubit in the circuit and that they could detect two distinct types of qubit errors. Previous efforts to achieve multiple error-correction rounds have only worked for one type. "Essentially, we have demonstrated for the first time all of the elements needed for a single [error-tolerant] logical qubit," says Ryan-Anderson.

"This is very high-caliber experimental work," says Steane. "It is one thing to deduce by analysis the effect of certain operations and measurements on qubits; it is quite another to achieve it in the lab," as the Honeywell team has now done. The work has "highlighted which parts of all the theoretical work on QEC and fault tolerance proved to be of practical use," says Steane.

"This is a significant advance," says Laird Egan, a quantum engineer at the Maryland-based company lonQ, which has previously implemented QEC methods for trapped-ion qubits. "What really distinguishes it is the multiple rounds of true fault-tolerant error correction"-something the IonQ team has not yet achieved.

QEC requires each logical qubit to be composed of multiple physical qubits, so the logical qubit incurs more potential sources of error than any of its component qubits. Ideally, the QEC can be fast enough that the logical qubit's error rate is lower than that of any physical qubit, but the Honeywell team's QEC, like all others developed so far, has not yet reached that level. So errors will still accumulate faster than they can be corrected. Reaching the so-called error-correction threshold, when QEC allows a logical qubit to outperform its physical qubits, "will be a big prize to whoever achieves it," Egan says. "I would not be surprised to see this threshold achieved in the next few years."

Philip Ball is a freelance science writer in London. His latest book is The Modern Myths (University of Chicago Press, 2021).

\section{REFERENCES}

1. C. Ryan-Anderson et al., "Realization of real-time fault-tolerant quantum error correction," Phys. Rev. X 11, 041058 (2021).

2. A. M. Steane, "Error correcting codes in quantum theory," Phys. Rev. Lett. 77, 793 (1996). 